\title{
Audio-Based Interventions in Sport
}

\author{
Fabrizio Sors ${ }^{1, *}$, Mauro Murgia ${ }^{1,2}$, Ilaria Santoro ${ }^{1}$ and Tiziano Agostini ${ }^{1}$ \\ ${ }^{I}$ Department of Life Sciences, University of Trieste, Italy; ${ }^{2}$ Department of Pedagogy, Psychology, Philosophy, University \\ of Cagliari, Italy
}

\begin{abstract}
In common practice, sports-perceptual interventions are mainly based on vision. However, research demonstrates that sporting performance can also be improved through the use of sounds, showing the relevance of the auditory channel to convey sports-related information, which can positively affect athletes' motor outcomes. This review examines the potential of audio-based interventions in sport. The relevant concepts are defined, a brief overview of the techniques based on vision is given and laboratory studies demonstrating the effectiveness of sounds in improving the execution of simple rhythmic motor tasks are reviewed. Subsequently, neurophysiological evidence of the influence of sounds on the motor regions of the brain is provided and different kinds of audio-based interventions, emphasising their methodological details and the effects of their application to specific sporting performances are described. Finally, recommendations for further research in this field, aimed both at maximizing the potential of audio-based interventions and their implementation at applied sporting contexts, are suggested.
\end{abstract}

Keywords: Audio-based interventions, augmented feedback, modeling, movement, perception, sound, sport.

\section{INTRODUCTION}

The aim of this review is to address audio-based interventions in sport, which represent a promising set of techniques that are not used at the moment in common practice. As a matter of fact, sports-perceptual interventions are traditionally based on vision: The correct execution of a given gesture/movement is shown either by the coach himself or through a video clip. It is also becoming more common to record an athlete's performance in order to analyse and "vivisect" it later. A clear-cut example of this practice is provided by track and field, where jumpers are recorded by their respective coaches, who then try to correct athletes' mistakes by giving them feedback on their performance while watching the clips. However, research shows that sporting accomplishments can also be improved through the use of sounds. Thus, the auditory channel has an important role in sports-perceptual interventions.

This review starts with a definition of the relevant concepts. Then, after a brief description of the various techniques based on vision, the focus switches to audition: As the first step, we describe the studies that highlight the effectiveness of sounds in improving the execution of simple rhythmic motor tasks. As the second step, we provide some neurophysiological evidence, which demonstrates that sound can spontaneously recruit motor regions of the brain. Subsequently, we describe different kinds of audio-based interventions and their application to specific sporting performances. Finally, we draw some conclusions and make recommendations to further develop this emerging field of research.

*Address correspondence to this author at the Department of Life Sciences, University of Trieste, Via Weiss 21 - Building W -34128 , Trieste (TS), Italy; Tel: +390405586182; Fax: +390405582134;

E-mail: fabrizio.sors@libero.it

\section{DEFINITIONS}

When dealing with motor improvement and learning, two fundamental concepts need to be taken into consideration: augmented feedback and modeling. According to Magill [1], augmented feedback refers to performance-related information, with the term "augmented" highlighting the fact of adding to or enhancing task-intrinsic feedback (which is the sensory-perceptual information that is a natural part of performing a skill). More specifically, Sigrist, Rauter, Riener \& Wolf [2] define augmented feedback as information that cannot be elaborated without an external source; thus it is provided by a coach or a display [3,4]. The term display is not limited to the visual modality (e.g. screens or projectors), but also refers to the auditory modality (e.g. headphones or speakers). Through these displays, augmented feedback provides knowledge of performance, that is, information regarding the current status of performance (i.e. what one is doing/has done right now).

Modeling has been defined as the use of demonstration as a means of conveying information about how to perform a skill [1]. According to a more general definition provided by APA, modeling is a process in which a person serves as a model for others, exhibiting the behavior to be imitated [5]. This concept can be extended, including the possibility that the person serving as a model can observe herself/himself at a different time. Dowrick [6] described self modeling as an intervention procedure using the observation of images of oneself (commonly captured on video) engaged in adaptive behavior. Like augmented feedback, modeling is not limited to the visual modality: Indeed, auditory models can be defined as sequences of sounds that reproduce different aspects of a given movement (e.g. timing, force, duration), thus representing an auditory form of demonstration. 
To sum up, augmented feedback provides athletes with additional information about their own performance, making them aware of pieces of information that are not available in normal conditions, and allowing them to use this new acquired information to adjust their own performance. Instead, modeling shows an ideal/optimal performance that athletes should try to imitate. The ideal performance can be executed either by another athlete or by the athlete himself (self modeling). In the latter case, the performance serving as a model would be her/his best performance. Conversely, the observation of the last executed one's own performance should be considered a case of augmented feedback.

In this review, we use the expression "audio-based intervention" to refer both to auditory augmented feedback and to auditory modeling, including both concepts under a single label. In particular, this expression refers to auditory stimuli that are informative about the execution of a movement, and thus are used to improve the movement itself and its outcome. An important distinction concerns the moment at which the auditory stimuli are provided. If the stimuli are provided during the execution of the movement (e.g. while rowing), they are defined as concurrent; however, if the stimuli are provided at the end of a trial, and before a new one in the case of repeated trials (e.g. between two subsequent rowing rides), they are defined as terminal (for an overview of related issues, see [1]). Another important point regards the duration of the intervention. Within the label of "audio-based intervention", we include both those interventions consisting of a single session and having an immediate effect, and those consisting of multiple sessions over a prolonged period of time and having both immediate and longterm effects.

Our definition of "audio-based intervention" does not include other interesting areas of research dealing with sports and sounds, such as music-based interventions [7, 8]. In sports, music is usually related to important variables influencing performances, such as psychophysiological arousal, mood and motivation, which are beyond the scope of the present review. Moreover, it is undeniable that for aesthetic sports (e.g. figure skating, rhythmic gymnastics, synchronized swimming, and so on) music represents a fundamental aspect, as athletes need to be "on time" while performing their movements. However, music does not directly convey any information about the movements' execution itself. For these reasons, the role of music will not be addressed in our work (for a review, see $[9,10]$ ).

\section{FROM VISUAL TO AUDIO-BASED INTERVEN- TIONS}

As previously stated, sports-perceptual interventions are mainly based on vision. The first practice mentioned in the introduction, i.e. the demonstration of the correct execution of a given gesture/movement, is a clear example of modeling. This pragmatic technique has its scientific foundation in Bandura's [11] research on imitation learning, as well as in other studies that investigated the learning of simple motor sequences through the exposition to visual models [12-16]. The rationale underlying this method is that an accurate observation of a correct movement can promote the emulation of that motor pattern, thus generating learning. From a neu- rophysiological perspective, this happens due to mirror neuron clusters, which discharge in motor areas even if the movement is only observed [17].

The second practice mentioned in the introduction, i.e. the recording and analysis of a given gesture/movement, is a clear example of augmented feedback. In the literature dealing with this technique, there is a strong debate concerning the combination of two parameters, i.e. task complexity and temporal characteristics of the feedback. Task complexity can be defined as the number of movement steps that are required to execute a task [18], while the temporal characteristics of the feedback refer to the above mentioned distinction between concurrent and terminal feedback. A metaanalysis by Marschall, Bund \& Wiemeyer [19] demonstrates that generally, the more complex the task, the more the trainee will profit from concurrent feedback.

Besides modeling and augmented feedback, another group of visual techniques aims to improve the perceptual processes that precede the execution of movements. These techniques concern visual occlusions - consisting of spatial and temporal occlusions - and eye-tracking. The use of these techniques, either alone or in combination, led to two important results: on the one hand, it has been found that anticipation, i.e. the ability to read and interpret the kinematic information present in the opponents' actions, is strongly associated with expertise [20-23]; on the other hand, it has been demonstrated that both novice and expert athletes can improve their ability to anticipate by training based on these techniques [24-28].

Although humans are mainly guided by visual information, we cannot ignore the relevance of other sources of information associated with human movement. Indeed, the information acquisition process is not limited to the visual domain, but includes information from the auditory, tactile and proprioceptive modalities. In sports-perceptual-motor research, the majority of studies focuses on the visual domain due to its dominance in humans (for a review, see [29]), while less attention is given to other sources of information. However, these sources provide precious information regarding the coordination and execution of movement, which might affect sporting performance. Among these sources, the auditory one deserves particular attention, since recent studies have shown very promising results, which are reviewed in this present work. In the following sections we address the role of audio-based interventions, starting with simple rhythmic movements and moving towards more complex, sports-related ones.

\section{AUDIO-BASED INTERVENTIONS FOR RHYTHMIC MOVEMENTS}

The effectiveness of audio-based interventions in promoting the acquisition and the correct reproduction of simple rhythmic movements is well established in literature. In a study, Doody, Bird \& Ross [30] asked participants to displace some padded wooden barriers in a predetermined sequence and time, a task that produced a distinct, auditory rhythmic pattern. Before performing this task, participants were exposed to either an auditory model, a visual model, an audiovisual model, or to no model at all (control condition). The results revealed that the critical factor in the accurate 
acquisition of the motor sequence was the presence of the auditory model, regardless of the presence or absence of the visual model. In a subsequent study, McCullagh \& Little [31] confirmed the superiority of audio and audiovisual models over visual ones, and highlighted the importance of knowledge of results in determining performance improvements.

The superiority of auditory models was also shown by Glenberg \& Jona [32], using a similar task (i.e. the reproduction of rhythmic sequences) and similar models (i.e. auditory and visual), while further manipulating another variable, the interstimulus interval. The results revealed that auditory models were more effective than visual ones, especially for short interstimulus intervals; but this superiority decreased proportionally with the increase of the intervals. According to the authors, this outcome was due to the fact that sequences with short intervals were identified like a rhythmic unit, while it was harder to identify units when the intervals were longer.

A fundamental concept when dealing with rhythmic movements is timing, which is the temporal structure of a movement. Research by Keele, Pokorny, Corcos \& Irvy [33] examined timing by studying the relationship between motor production timing and perceptual timing in the auditory modality. To measure motor production timing, subjects were asked to synchronize their tapping on a key with a regular click. After a defined time, the click stopped and participants were required to keep tapping, following the same rhythm. The measure of interest was the standard deviation of the intertap intervals after the click stopped. To measure perceptual timing, subjects were asked to judge whether an interval between two clicks was longer or shorter than the standard interval heard a second before. The measure of interest was the difference between the upper and lower thresholds obtained through a series of such judgments. The results revealed that the two measures were significantly correlated, leading the authors to claim that the mechanisms underlying motor production timing and perceptual timing are closely interconnected.

Lai, Shea \& Little [34] described another important distinction that characterizes timing, i.e. absolute and relative timing. Absolute timing is "the manner in which a movement sequence, as a whole, is carried out, without regard to individual components of the movement" [34, p350]. Relative timing is "the degree to which the proportion of time between movement segments (segment movement time-total movement time) is achieved without regard to the overall (absolute) time used" [34, p350]. A study of Shea, Wulf, Park \& Gaunt [35] investigated separately the effects of auditory models on absolute and relative training. The participants were randomly assigned to either an auditory model or a control condition (absence of model). Another variable manipulated by the authors was the physical practice with the rhythmic task. Within the auditory/control conditions, in the acquisition phase participants were coupled, so that in each couple one participant practiced the task directly, while the other one only observed him/her. However, twenty-four hours later, in the retention phase, all participants were asked to perform the task. The results revealed that participants who experienced the auditory model in the acquisition phase, regardless of physical practice, showed a more accurate rela- tive timing compared to those who were in the control group; however, absolute timing was more accurate for participants who physically practiced the task, and even more for those who experienced the auditory model. These results highlight that for the improvement of both absolute and relative timing it is not sufficient to passively listen to an auditory model, rather it is necessary to physically practice the specific task.

Beside the studies described here, other research demonstrates that audio-based interventions are more effective than visual ones in promoting the identification, discrimination, memorization and reproduction of simple, precisely timed movements [36-44]. Altogether, these studies suggest that the auditory system is more pertinent than the visual one to take advantage of the rhythmic features of simple movements; which, in turn, promotes the accurate reproduction of the movements themselves.

\section{NEUROPHYSIOLOGICAL EVIDENCE}

Among the authors mentioned in the previous section, Keele et al. [33] claimed that there is a tight synergy between the mechanisms underlying auditory perceptual timing and motor production timing. In subsequent years, their claim gained support through neurophysiological research, in particular by Chen, Penhune \& Zatorre [45]. In their first experiment, participants were required to listen to rhythmic sequences of sounds, being aware that they would have to reproduce the same rhythms by tapping later. In their second experiment, a different group of participants listened to the same rhythms as in the first experiment, but without foreknowledge that they would have to reproduce them later. Functional magnetic resonance imaging (fMRI) was used to monitor the participants' brain activity. The results revealed that, besides the recruitment of other areas, the supplementary motor area, the mid-premotor cortex and the cerebellum were activated during the listening phase of both experiments. This outcome shows that listening to rhythmic sequences of sound promotes the activation of motor regions in the brain. This also happens when there is no knowledge that a motor task related to those sounds will have to be performed later. Similar results were obtained in numerous other studies [46-50], thus confirming a close connection between auditory perception and motor production.

The above mentioned studies related to rhythmic sequences. Other studies researched more complex sounds, i.e. those deriving from specific movements. In the section related to visual interventions, mirror neurons were mentioned, since they were first discovered for the visual modality [17]. However, it was discovered that the mirror neuron system is also sensitive to auditory stimuli. Indeed, these neurons do not only discharge if an action is performed or seen, but also when the corresponding action-related sounds are heard [51, 52]. Pizzamiglio et al. [53] demonstrated that the sounds of actions, in particular those produced by human movements, activate human motor and pre-motor areas, whereas sounds of a different nature, such as noise and environmental sounds, do not.

Various studies confirm that movement-related sounds promote the activation of pre-motor and motor brain areas $[54,55]$. Two studies deserve particular attention, since they deal with sports-related sounds. The first one is by Woods, 
Hernandez, Wagner \& Beilock [56], in which fMRI was used to monitor the brain activity of expert and novice athletes in response to familiar and unfamiliar, sports and nonsports sounds. In particular, participants played basketball or tennis either at college varsity or at recreational level, and were considered experts or novices, respectively. The sports sounds consisted of sounds from basketball and tennis, including five variations of a basketball bouncing on a court, and five variations of a tennis ball being hit by a racquet; non-sports sounds were composed of common environmental sounds such as a toilet flushing or paper crumpling. For sports sounds, familiarity depended on the sport that participants played, while for the non-sports sounds the distinction was based on the identifiability of the sound, rather than its commonality. The results not only confirmed that movement-related sounds promote the activation of premotor and motor brain areas, but also highlighted an expertise effect: experts showed greater activation than novices during the sports familiar condition.

The second study was by Schmitz et al. [57]; the sounds used as stimuli were generated according to the movement sonification technique. This technique consists of the conversion of physical and/or kinematic parameters of the movement under investigation into a synthetic sound, which conveys meaningful information about the variation of those parameters $[58,59]$. In the study participants were exposed to audiovisual sonified clips of a solid computer-generated swimmer model performing breaststroke movements. The auditory information consisted of synthetic sounds, whose parameters varied as a function of various kinematic parameters of the model. In one condition the sounds matched the visual movement kinematics (congruent condition), while in the other there was no correspondence between the sound and movement kinematics (incongruent condition). The fMRI results revealed greater brain activation in the premotor and motor areas in the congruent condition than in the incongruent condition. This outcome highlights that, besides movement-related sounds, synthetic sound information about the execution of a movement promotes the activation of motor areas in the brain as well.

Altogether, these studies demonstrate that listening to both simple rhythmic sequences and more complex movement-related sounds promotes the activation of pre-motor and motor regions of the brain, even when no action is required. This fact highlights a close connection between auditory perception and motor production: the next two sections describe how this connection has been exploited to promote performance improvements in sport.

\section{AUDIO-BASED INTERVENTIONS IN SPORT: AUDI- TORY AUGMENTED FEEDBACK}

As described above, synthetic sounds that are informative about the execution of a movement directly influence the motor areas of the brain. Other studies explored whether these kinds of stimuli, in the form of augmented feedback, can also affect motor performance. The first sport considered was swimming, where studies demonstrated that various kinds of concurrent sonification promoted significant improvements in the crawl performance of expert swimmers $[60,61]$. Subsequently, in a study by Hummel, Hermann,
Frauenberger \& Stockman [62], the effects of concurrent sonification of the German wheel rolling motion were compared between novices and experts. The results suggested that only experts benefitted from the auditory stimulus by significantly improving the execution of basic moves.

Other sports, such as karate [63] and skiing [64], were also considered; however, in both cases the researchers did not measure objective performance variables. Instead, athletes' subjective evaluations of the potential effectiveness of sonification in improving their own performances were assessed, obtaining encouraging results.

In recent years, a sport receiving particular attention is rowing, since it is characterized by the complex rhythmic movement of the rowing cycle. Two perceptual studies showed that rowers were able both to recognize the rowing cycle through different kinds of sonification [65], and to identify their own amongst those of other athletes [66]. Moreover, it has been demonstrated that expert rowers benefit from concurrent sonification in terms of performance improvement. Schaffert, Mattes \& Effenberg [67] developed a device that sonified online the acceleration of rowing boats, with tone pitch increasing as the boat acceleration increases, and tone pitch decreasing as boat acceleration decreases. Such a device provides rowers with a concurrent auditory augmented feedback, giving them the possibility to monitor the effectiveness of their rowing cycle online. The results revealed that, at the same stroke rate, boat velocity was significantly higher when the sonification system was switched on than when it was switched off. Moreover, the distance travelled, a factor dependent on the boat velocity, was also greater when the sonification system was switched on than when it was switched off. Later, encouraging results were obtained from adaptive athletes (both with physical and visual disabilities) [68], as well as from testing various kinds of sonification obtained by using different criteria for the conversion of boat acceleration into sound [69].

Finally, in a recent study Kennel et al. [70] compared the effects of concurrent auditory feedback, delayed (180ms) auditory feedback and white noise on hurdling performances. The results revealed that delayed auditory feedback significantly decreased performance by slowing down the time to complete the track. However, no differences were found between the real-time auditory feedback condition and the white noise condition.

\section{AUDIO-BASED INTERVENTIONS IN SPORT: AUDI- TORY MODELING}

In the section about neurophysiological evidence, the studies showed that listening to movement-related sounds promoted the activation of pre-motor and motor regions of the brain [53-56]. Other studies tested whether athletes were able to identify the sound produced by their own performance among those of other athletes. In particular, Murgia, Hohmann, Galmonte, Raab \& Agostini [71] pursued this aim in golf by audio-recording the swing of different expert golfers. Participants were exposed to sounds associated both with their own performance and with those of other golfers. Using a two alternative forced choice paradigm, participants were required to state whether each sound was theirs or another golfer's. The results revealed that athletes were able to 
correctly recognise their own swing through sound, and that a crucial factor for recognition was the temporal structure of the stimuli. The ability to identify the sounds associated with one's own performance was also demonstrated in hurdling $[72,73]$ and received further support through neurophysiological evidence that listening to one's own long jumps versus those of other athletes promoted the activation of different brain areas [74].

While from a neurophysiological perspective, these results can be explained in terms of audiovisual mirror neurons $[51,52]$, from a theoretical perspective, they can be explained by the Theory of Event Coding (TEC) [75, 76], which assumes that perception and action share a common representational system. According to the TEC, the match between the feature codes elicited by sounds associated with movements and the feature codes pre-activated in the representational system (due to motor experience) result in a better quality for one's own sounds, compared to the sounds of others. As a consequence, a higher level of matching generates a synergic activation of those codes, determining their binding. The binding of the most activated codes facilitate the recognition of the performer (self versus others).

The assumption that perception and action share a common representational system allows us to make predictions in the opposite direction, as perceptual processes activate the codes associated with the features of perceived events. These feature codes are pre-activated when people have to make a movement and, because of this activation, these codes have a higher probability to be used in motor production. In the case of movement-related sounds, listening to them promotes the activation of the codes associated with the features of those sounds (and of the movements they represent). Moreover, the codes activated by these sounds match the codes preactivated by previous motor experience, with the result that these codes prime each other on the basis of their overlap. As a consequence, the execution of a movement is affected by the synergic activation of the codes activated by both movement-related sounds and previous motor experience, with an increased probability for those feature codes to be retrieved by the motor system and consequently to be performed, as suggested by Murgia et al. [77]. Furthermore, the effects of movement-related sounds on motor systems increase as a function of the motor experience. The synergic activation of the feature codes due to both auditory stimuli and motor experience - regarding a given movement - should determine a greater activation of feature codes in experts, compared to novices, in performing that specific movement.

A number of studies seem to corroborate these predictions by demonstrating the effectiveness of auditory modeling to improve sporting performance. Agostini, Righi, Galmonte \& Bruno [78] investigated hammer throw. On the first day, participants, who were expert throwers, were required to perform two series of ten throws each, in order to control for the fatigue effect. On the second day, participants performed again two series of ten throws each. During the first series the authors recorded the sound produced by the hammer's friction with the air while rotating, by placing a microphone on the hammer head; the first series also served as a baseline. The second series was the experimental session: The sound associated with the longest baseline throw of each athlete was used as an auditory model and presented five times before each throw. Two kinds of performance improvements were obtained: The experimental throws showed both a significant increase in average length and a significant decrease in length variability, compared to the baseline.

Similar upward standardizations of key aspects of swimming [79] and soccer free kick [80] performances were also obtained, by adapting the described modus operandi to the specific characteristics of the sport. In particular, as concerns swimming [79], an ideal model was created by looping the sound of the most effective stroke cycle, which was chosen by a swimmer together with his coach. As concerns soccer [80], authors used as a model the run up of the best free kick obtained by each participant during a baseline session. The best performances were selected using both subjective (i.e., self-rating) and objective parameters (i.e., the kicks that entered the goal nearer to the top corner behind the wall).

Other examples of the effectiveness of auditory modeling are provided by studies on weightlifting [81] and skateboarding [82]. In particular, as concerns weightlifting, Murgia et al. [81] created auditory models to guide expert lifters during the one-repetition bench press exercise. These models consisted of an initial countdown, followed by a low-intensity sound, which corresponded to the down phase of the exercise, and then by a high-intensity sound, which corresponded to the pressing phase. The results revealed that the average power exerted in the auditory stimulation condition was significantly greater than that exerted in the control condition. As concerns skateboarding, Cesari, Camponogara, Papetti, Rocchesso \& Fontana [82] demonstrated that listening to synthetic sounds simulating the run of a skateboard promotes the activation of leg muscles. In particular, the activation patterns of experts, compared to those of novices, closely resembled the activation patterns needed to actually perform the various events that occur during skateboarding (acceleration, steady run, deceleration, and jump). These results suggest that familiarity is needed to correctly interpret specific sounds.

Other studies compared the effectiveness of different type of models. For instance, Effenberg [59] sonified countermovement jumps by mapping the vertical component of the ground reaction force, measured by a force plate, to the amplitude and the frequency of sound as an electronically sampled vocal $a$. Sports students were then asked to reproduce as accurately as possible the height of jumps in two conditions: one, after watching a mute video clip (visual model) or two, after watching a sonified video clip (audiovisual model). Participants were significantly more accurate in the latter condition. In another study by Ramezanzade, Abdoli, Farsi \& Sanjari [83] the angular speed of the elbow joint of a professional basketball player performing jump shots was sonified. Participants, who were novices to basketball, were exposed either to a mute video clip (visual model) or to a sonified video clip (audiovisual model); those experiencing the latter clip provided significantly better performances. The authors of both studies explained their results in terms of multisensory integration, but the absence of an audio-only condition did not allow disentangling the relative "weight" of auditory information in comparison with visual information. 
A possible answer to this question can be found in a study by Murgia et al. [84], who demonstrated that an auditory model is more effective than a visual one in improving the golfing performance of expert golfers, both in terms of performance outcomes (distance of shots from the target) and of movement execution (standardization of both relative timing and overall duration of the swing). Similar results had been previously obtained by Righi, Ferletic, Furlan, Pin \& Gherzil [85] with young tennis players on the serve, with the important addition that the improvements promoted by the auditory model were greater than those promoted by the audiovisual one.

Altogether, the studies described in the last two sections demonstrate that audio-based interventions are beneficial not only for simple rhythmic movements, but also for the complex ones that characterize sports, promoting significant improvements in athletes' performances.

\section{CONCLUSION}

From this review emerges the potential of audio-based interventions to promote significant improvements in sporting performances as encouraging results have been obtained in different sports and with different types of protocols. However, besides continuing to test the effectiveness of these kinds of interventions in sports in which their effects are not yet proven, other research can be done in this field. In particular, future studies should focus on two areas: the first one concerns the nature of the interventions, i.e. their type (augmented feedback versus modeling) and the auditory stimuli used; the second area concerns the implementation of the interventions into applied sporting contexts.

Regarding the first area, it is fundamental to determine the protocols that maximize the potential of audio-based interventions. For auditory augmented feedback, the effectiveness of various kinds of sonification (for a review, see [86]) need to be compared, in order to identify both the most relevant parameters and the most effective ways to convert them into meaningful sounds. Moreover, the effects of auditory stimuli different from sonification should be better clarified, as well as it could be investigated the effectiveness of terminal augmented feedback. With regard to auditory modeling, a key challenge for future studies is to identify the most meaningful sounds to be given to athletes as a model; for example, it would be interesting to place the microphone(s) near the athletes' ears to test if such a model would be more effective than those deriving from the equipment perspective (e.g. the hammer head) or from an external one (e.g. the poolside). After having addressed the potential of auditory augmented feedback and auditory modeling separately, they should be compared in order to find out whether the benefits deriving from their use are similar or whether the different types of intervention are more or less effective depending on the specific sport under investigation.

Regarding the second area, future studies should clarify certain issues in the application of audio-based interventions into applied sporting contexts. One of the first issues concerns the effectiveness of these interventions in promoting skills acquisition in sport. Until now, studies have maintained that audio-based interventions are effective for performance enhancement, that is, for improving already ac- quired skills. However, the reviewed laboratory studies have demonstrated that audio-based interventions are also effective in promoting the acquisition of rhythmic tasks; moreover, there is a vast literature demonstrating the effectiveness of visual interventions in promoting the acquisition of sporting skills (for an overview, see [1]). As a consequence, it would be interesting to find out whether audio-based interventions could promote such an acquisition; and, if that is the case, comparisons could be drawn between visual and audio-based interventions in order to identify which athletic/technical gestures/movements are better and/or faster acquired through the former or the latter type of intervention.

A partially related issue concerns the relationship between expertise and audio-based interventions. According to our knowledge, only one study [62] compared the effects of an audio-based intervention between novices and experts: in that specific case, concurrent augmented feedback was beneficial for experts but not for novices. However, in another study [83] novices benefitted from an audiovisual model. This suggests that sound, in this case combined with visual information, can be beneficial also for inexperienced individuals. The role of auditory information in the acquisition of new sport skills is quite unexplored in literature, then future studies should further clarify whether such information can be effectively used with novice athletes.

Another important aspect to be clarified is whether the beneficial effects of audio-based interventions are long-term or not. Indeed, the majority of the studies described in the present review concerns interventions consisting of a single session and promoting immediate improvements. Future studies should test whether interventions consisting of multiple sessions over a prolonged period of time promote longlasting improvements in sporting performance.

Once these issues have been successfully investigated, it would be possible to integrate audio-based interventions into the training regimes of athletes and teams, diminishing the risk that the potential of such interventions remains only a matter of research and does not become a precious resource for applied sporting contexts.

\section{CONFLICT OF INTEREST}

The authors confirm that this article content has no conflict of interest.

\section{ACKNOWLEDGEMENTS}

The author Mauro Murgia was supported by Autonomous Region of Sardinia, Master \& Back Programme 2013 (PRRMAB-A2013-19330).

\section{REFERENCES}

[1] Magill R. Motor learning and control: concepts and applications. $9^{\text {th }}$ ed. New York, NY: McGraw-Hill 2010.

[2] Sigrist R, Rauter G, Riener R, et al. Augmented visual, auditory, haptic, and multimodal feedback in motor learning: a review. Psychon Bull Rev 2013; 20: 21-53.

[3] Schmidt RA, Wrisberg CA. Motor learning and performance: A situation-based learning approach. Champaign, IL: Human Kinetics 2008.

[4] Utley A, Astill S. Instant notes in motor control, learning and development. $1^{\text {st }}$ ed. New York: Taylor \& Francis 2008. 
[5] VandenBos GR. APA dictionary of psychology. Washington, DC, US: American Psychological Association 2007.

[6] Dowrick PW. A review of self modeling and related interventions. Appl Prev Psychol 1999; 8: 23-39.

[7] Karageorghis CI, Jones L. On the stability and relevance of the exercise heart rate-music-tempo preference relationship. Psychol Sport Exerc 2014; 15: 299-310.

[8] Sanchez X, Moss SL, Twist C, et al. On the role of lyrics in the music-exercise performance relationship. Psychol Sport Exerc 2014; 15: 132-8

[9] Karageorghis CI, Priest D-L. Music in the exercise domain: a review and synthesis (Part I). Int Rev Sport Exerc Psychol 2012; 5: 44-66.

[10] Karageorghis CI, Priest D-L. Music in the exercise domain: a review and synthesis (Part II). Int Rev Sport Exerc Psychol 2012; 5: 67-84.

[11] Bandura A. Social learning theory. Oxford, England: Prentice-Hall 1977.

[12] Blandin Y, Proteau L, Alain C. On the cognitive processes underlying contextual interference and observational learning. J Mot Behav 1994; 26: 18-26.

[13] McCullagh P, Stiehl J, Weiss MR. Developmental modeling effects on the quantitative and qualitative aspects of motor performance. Res Q Exerc Sport 1990; 61: 344-50.

[14] McCullagh P, Weiss MR, Ross D. Modeling considerations in motor skill acquisition and performance: An integrated approach. Exerc Sport Sci Rev 1989; 17: 475-514.

[15] Scully DM, Newell KM. Observational learning and acquisition of motor skills: Toward a visual perception perspective. J Hum Mov Stud 1985; 11: 169-86.

[16] Shea CH, Wright DL, Wulf G, et al. Physical and observational practice afford unique learning opportunities. J Mot Behav 2000; 32: $27-36$.

[17] Rizzolatti G, Fadiga L, Gallese V, et al. Premotor cortex and the recognition of motor actions. Cogn Brain Res 1996; 3: 131-41.

[18] Barris R, Kielhofner G, Levine RE, et al. Occupation as interaction with the environment. In: Kielhofner G, Ed. A model of human occupation: Theory and application. Baltimore: Williams \& Wilkins 1985; pp. 42-62.

[19] Marschall F, Bund A, Wiemeyer J. Does frequent feedback really degrade learning? a meta analysis. E-J Beweg Train 2007; 1: 75-86.

[20] Abernethy B, Gill DP, Parks SL, et al. Expertise and the perception of kinematic and situational probability information. Perception 2001; 30: 233-52.

[21] Abernethy B, Russell DG. Expert-novice differences in an applied selective attention task. J Sport Psychol 1987; 9: 326-45.

[22] Mann DTY, Williams AM, Ward P, et al. Perceptual-cognitive expertise in sport: a meta-analysis. J Sport Exerc Psychol 2007; 29: 457-78.

[23] Shim J, Carlton LG, Chow JW, et al. The use of anticipatory visual cues by highly skilled tennis players. J Mot Behav 2005; 37: 16475.

[24] Abernethy B, Wood JM, Parks S. Can the anticipatory skills of experts be learned by novices? Res Q Exerc Sport 1999; 70: 313-8.

[25] Farrow D, Abernethy B. Can anticipatory skills be learned through implicit video based perceptual training? J Sports Sci 2002; 20: 471-85.

[26] Hopwood M, Mann D, Farrow D, et al. Does visual-perceptual training augment the fielding performance of skilled cricketers? Int J Sports Sci Coach 2011; 6: 523-36.

[27] Murgia M, Sors F, Muroni AF, et al. Using perceptual hometraining to improve anticipation skills of soccer goalkeepers. Psychol Sport Exerc 2014; 15: 642-8.

[28] Ryu D, Kim S, Abernethy B, et al. Guiding attention aids the acquisition of anticipatory skill in novice soccer goalkeepers. Res Q Exerc Sport 2013; 84: 252-62

[29] Ste-Marie DM, Law B, Rymal AM, et al. Observation interventions for motor skill learning and performance: an applied model for the use of observation. Int Rev Sport Exerc Psychol 2012; 5: 145-76.

[30] Doody SG, Bird AM, Ross D. The effect of auditory and visual models on acquisition of a timing task. Hum Mov Sci 1985; 4: 2710-81.

[31] McCullagh P, Little WS. A comparison of modalities in modeling. Hum Perform 1989; 2: 101-11.
[32] Glenberg AM, Jona M. Temporal coding in rhythm tasks revealed by modality effects. Mem Cognit 1991; 19: 514-22.

[33] Keele SW, Pokorny RA, Corcos DM, et al. Do perception and motor production share common timing mechanisms: a correlational analysis. Acta Psychol 1985; 60: 173-91.

[34] Lai Q, Shea CH, Little M. Effects of modeled auditory information on a sequential timing task. Res Q Exerc Sport 2000; 71: 349-56.

[35] Shea CH, Wulf G, Park J-H, et al. Effects of an auditory model on the learning of relative and absolute timing. J Mot Behav 2001; 33: 127-38.

[36] Collier GL, Logan G. Modality differences in short-term memory for rhythms. Mem Cognit 2000; 28: 529-38.

[37] Glenberg AM, Mann S, Altman L, et al. Modality effects in the coding reproduction of rhythms. Mem Cognit 1989; 17: 373-83.

[38] Glenberg AM, Swanson NG. A temporal distinctiveness theory of recency and modality effects. J Exp Psychol Learn Mem Cogn 1986; 12: 3-15.

[39] Grondin S, McAuley JD. Duration discrimination in crossmodal sequences. Perception 2009; 38: 1542-59.

[40] Han D-W, Shea CH. Auditory Model: Effects on learning under blocked and random practice schedules. Res Q Exerc Sport 2008; 79: 476-86.

[41] Kohl RM, Shea CH. Augmenting motor responses with auditory information: guidance hypothesis implications. Hum Perform 1995; 8: 327-43.

[42] Lai Q, Shea CH, Bruechert L, et al. Auditory model enhances relative-timing learning. J Mot Behav 2002; 34: 299-307.

[43] Repp BH, Penel A. Auditory dominance in temporal processing: New evidence from synchronization with simultaneous visual and auditory sequences. J Exp Psychol Hum Percept Perform 2002; 28: 1085-99.

[44] Repp BH, Penel A. Rhythmic movement is attracted more strongly to auditory than to visual rhythms. Psychol Res 2004; 68: 252-70.

[45] Chen JL, Penhune VB, Zatorre RJ. Listening to musical rhythms recruits motor regions of the brain. Cereb Cortex 2008; 18: 284454.

[46] Bangert M, Altenmüller EO. Mapping perception to action in piano practice: a longitudinal DC-EEG study. BMC Neurosci 2003; 4: 26.

[47] Bengtsson SL, Ullén F, Ehrsson HH, et al. Listening to rhythms activates motor and premotor cortices. Cortex 2009; 45: 62-71.

[48] Fujioka T, Trainor LJ, Large EW, et al. Internalized timing of isochronous sounds is represented in neuromagnetic beta oscillations. J Neurosci 2012; 32: 1791-802.

[49] Grahn JA, Brett M. Rhythm and beat perception in motor areas of the brain. J Cogn Neurosci 2007; 19: 893-906.

[50] Schubotz RI, Friederici AD, von Cramon DY. Time perception and motor timing: A common cortical and subcortical basis revealed by fMRI. NeuroImage 2000; 11: 1-12.

[51] Keysers C, Kohler E, Umiltà MA, et al. Audiovisual mirror neurons and action recognition. Exp Brain Res 2003; 153: 628-36.

[52] Kohler E, Keysers C, Umiltà MA, et al. Hearing sounds, understanding actions: Action representation in mirror neurons. Science 2002; 297: 846-8.

[53] Pizzamiglio L, Aprile T, Spitoni G, et al. Separate neural systems for processing action- or non-action-related sounds. NeuroImage 2005; 24: 852-61.

[54] Aziz-Zadeh L, Iacoboni M, Zaidel E, et al. Left hemisphere motor facilitation in response to manual action sounds. Eur J Neurosci 2004; 19: 2609-12.

[55] Gazzola V, Aziz-Zadeh L, Keysers C. Empathy and the somatotopic auditory mirror system in humans. Curr Biol 2006; 16: 18249.

[56] Woods EA, Hernandez AE, Wagner VE, et al. Expert athletes activate somatosensory and motor planning regions of the brain when passively listening to familiar sports sounds. Brain Cogn 2014; 87: 122-33.

[57] Schmitz G, Mohammadi B, Hammer A, et al. Observation of sonified movements engages a basal ganglia frontocortical network. BMC Neurosci 2013; 14: 32.

[58] Effenberg AO. Sonification: ein akustisches Informationskonzept zur menschlichen Bewegung. Schorndorf: Hofmann 1996.

[59] Effenberg AO. Movement sonification: effects on perception and action. IEEE Multimed 2005; 12: 53-9.

[60] Chollet D, Madani M, Micallef JP. Effects of two types of biomechanical bio-feedback on crawl performance. In: MacLaren D, 
Reilly T, Lees A, Eds. Biomechanics and Medicine in Swimming, Swimming Science VI. London: E \& F Spon 1992; pp. 48-53.

[61] Chollet D, Micallef JP, Rabischong P. Biomechanical signals for external biofeedback to improve swimming techniques. In: Ungerechts BE, Wilke K, Reishle K, Eds. Swimming science V. Champaign: Human Kinetics Publishers 1988; pp. 389-96.

[62] Hummel J, Hermann T, Frauenberger C, et al. Interactive sonification of German wheel sports movement. In: Proceedings of ISon 2010, $3^{\text {rd }}$ Interactive Sonification Workshop 2010; pp. 17-22.

[63] Yamamoto G, Shiraki K, Takahata M, et al. Multimodal knowledge for designing new sound environments. In: Workshop on Mobile HCI and Sound 2004; pp. 31-6.

[64] Kirby R. Development of a real-time performance measurement and feedback system for alpine skiers. Sports Technol 2009; 2: 4352 .

[65] Dubus G. Evaluation of four models for the sonification of elite rowing. J Multimodal User Interfaces 2012; 5: 143-56.

[66] Schmitz G, Effenberg AO. Perceptual effects of auditory information about own and other movements. In: Proceedings of the $18^{\text {th }}$ International Conference on Auditory Display 2012; pp. 89-94.

[67] Schaffert N, Mattes K, Effenberg AO. An investigation of online acoustic information for elite rowers in on-water training conditions. J Hum Sport Exerc 2011; 6: 392-405.

[68] Schaffert N, Mattes K. Acoustic feedback training in adaptive rowing. In: Proceedings of the $18^{\text {th }}$ International Conference on Auditory Display 2012; pp. 83-8.

[69] Schaffert N, Gehret R. Testing different versions of functional sonification as acoustic feedback for rowing. In: Proceedings of The $19^{\text {th }}$ International Conference on Auditory Display 2013; pp. 331-5.

[70] Kennel C, Streese L, Pizzera A, et al. Auditory reafferences: The influence of real-time feedback on movement control. Front Psychol 2015; 6: 69.

[71] Murgia M, Hohmann T, Galmonte A, et al. Recognising one's own motor actions through sound: the role of temporal factors. Perception 2012; 41: 976.

[72] Kennel C, Hohmann T, Raab M. Action perception via auditory information: Agent identification and discrimination with complex movement sounds. J Cogn Psychol 2014; 26: 157-65.

[73] Kennel C, Pizzera A, Hohmann T, et al. The perception of natural and modulated movement sounds. Perception 2014; 43: 796-804.
[74] Justen C, Herbert C, Werner K, et al. Self vs. other: Neural correlates underlying agent identification based on unimodal auditory information as revealed by electrotomography (sLORETA). Neuroscience $2014 ; 259: 25-34$.

[75] Hommel B, Müsseler J, Aschersleben G, et al. The Theory of Event Coding (TEC): A framework for perception and action planning. Behav Brain Sci 2001; 24: 849-78.

[76] Zmigrod S, Hommel B. Auditory event files: Integrating auditory perception and action planning. Atten Percept Psychophys 2009; 71: 352-62.

[77] Murgia M, Santoro I, Tamburini G, et al. Ecological sounds affect breath duration more than artificial sounds. Psychol Res 2015. [epub ahead of print]. DOI 10.1007/s00426-015-0647-z.

[78] Agostini T, Righi G, Galmonte A, et al. The relevance of auditory information in optimizing hammer throwers performance. In: Pascolo PB, Ed. Biomechanics and Sports. Vienna, Austria: Springer 2004; pp. 67-74.

[79] Galmonte A, Righi G, Agostini T. Stimoli acustici come nuovo elemento per il miglioramento della performance nel nuoto. Movimento 2004; 20: 73-8.

[80] Prpic V, Murgia M, Feduzi S, et al. Modelli acustici applicati ai calci di punizione nel gioco del calcio. Movimento 2010; 26: 74-8.

[81] Murgia M, Sors F, Vono R, et al. Using auditory stimulation to enhance athletes' strength: An experimental study in weightlifting. Rev Psychol 2012; 19: 13-6.

[82] Cesari P, Camponogara I, Papetti S, et al. Might as well jump: sound affects muscle activation in skateboarding. PLoS One 2014; 9: e90156.

[83] Ramezanzade H, Abdoli B, Farsi A, et al. The effect of sonification modelling on perception and accuracy of performing jump shot basketball. Int J Sport Stud 2014; 4: 1388-92.

[84] Murgia M, Bresolin G, Righi G, et al. The effect of visual and auditory models on golf swing. J Sport Exerc Psychol 2011; 33: s91.

[85] Righi G, Ferletic E, Furlan D, et al. Are visual models the best models to learn a specific task in sport training? [abstract] Perception 2007; 36(1 Suppl): 179.

[86] Dubus G, Bresin R. A systematic review of mapping strategies for the sonification of physical quantities. PLoS One 2013; 8: e82491.

(C) Sors et al.; Licensee Bentham Open.

This is an open access articles licensed under the terms of the Creative Commons Attribution-Non-Commercial 4.0 International Public License (CC BY-NC 4.0) (https://creativecommons.org/licenses/by-nc/4.0/legalcode), which permits unrestricted, non-commercial use, distribution and reproduction in any medium, provided that the work is properly cited. 